\title{
Necessary Optimality Conditions for a Dead Oil Isotherm Optimal Control Problem*
}

\author{
Moulay Rchid Sidi Ammi ${ }^{\dagger} \quad$ Delfim F. M. Torres ${ }^{\ddagger}$
}

\begin{abstract}
We study a system of nonlinear partial differential equations resulting from the traditional modelling of oil engineering within the framework of the mechanics of a continuous medium. Recent results on the problem provide existence, uniqueness and regularity of the optimal solution. Here we obtain the first necessary optimality conditions.
\end{abstract}

Mathematics Subject Classification 2000: 49K20, 35K55.

Key Words: optimal control of distributed systems; dead oil isotherm problem; necessary optimality conditions.

\section{Introduction}

We are interested in the optimal control of the dead oil isotherm problem:

$$
\begin{cases}\partial_{t} u-\Delta \varphi(u)=\operatorname{div}(g(u) \nabla p) & \text { in } Q_{T}=\Omega \times(0, T), \\ \partial_{t} p-\operatorname{div}(d(u) \nabla p)=f & \text { in } Q_{T}=\Omega \times(0, T), \\ \left.u\right|_{\partial \Omega}=0,\left.\quad u\right|_{t=0}=u_{0}, & \\ \left.p\right|_{\partial \Omega}=0,\left.\quad p\right|_{t=0}=p_{0}, & \end{cases}
$$

where $\Omega$ is an open bounded domain in $\mathbb{R}^{2}$ with a sufficiently smooth boundary. Equations (11) serve as a model for an incompressible biphasic flow in a porous medium, with applications to the industry of exploitation of hydrocarbons. To understand the optimal control problem we will consider here, some words about the recovery of hydrocarbons are in order. At the time of the first

\footnotetext{
*Work supported by the Portuguese Foundation for Science and Technology (FCT) through the Centre for Research in Optimization and Control (CEOC) of the University of Aveiro, cofinanced by the European Community fund FEDER/POCTI. The first author was also supported by the postdoc fellowship SFRH/BPD/20934/2004. Research Report CM06/I-42.

${ }^{\dagger}$ Postdoc Researcher, Department of Mathematics, University of Aveiro, 3810-193 Aveiro, Portugal. Email: sidiammi@mat.ua.pt

¥Associate Professor, Department of Mathematics, University of Aveiro, 3810-193 Aveiro, Portugal. Email: delfim@mat.ua.pt
} 
run of a layer, the flow of the crude oil towards the surface is due to the energy stored in the gases under pressure in the natural hydraulic system. To mitigate the consecutive decline of production and the decomposition of the site, water injections are carried out, well before the normal exhaustion of the layer. The water is injected through wells with high pressure, by pumps specially drilled to this end. The pumps allow the displacement of the crude oil towards the wells of production. The wells must be judiciously distributed, which gives rise to a difficult problem of optimal control: how to choose the best installation sites of the production wells? This is precisely the question we address in this work: our main goal is to present a method to carry out the optimal control of (11) with respect to all the important parameters that intervene in the process. More precisely, we seek necessary conditions for the admissible parameters $u, p$ and $f$ to minimize the functional

$$
J(u, p, f)=\frac{1}{2}\|u-U\|_{2, Q_{T}}^{2}+\frac{1}{2}\|p-P\|_{2, Q_{T}}^{2}+\frac{\beta_{1}}{2}\|f\|_{2 q_{0}, Q_{T}}^{2 q_{0}}+\frac{\beta_{2}}{2}\left\|\partial_{t} f\right\|_{2, Q_{T}}^{2}
$$

where $q_{0}>1$ and $\beta_{1}>0$ and $\beta_{2}>0$ are two coefficients of penalization. The first two terms in (2) make possible to minimize the difference between the reduced saturation of oil $u$, the global pressure $p$ and the given data $U$ and $P$.

Existence and uniqueness to the system (11), for the case when the term $\partial_{t} p$ is missing but for more general boundary conditions, is established in [1]. In [2] we obtain conditions which provide existence and regularity of the optimal solutions to the problem of minimizing (2) subject to (11). Here we are interested to obtain necessary optimality conditions which permit to find the solutions predicted by the results in [2]. This is, to the best of our knowledge, an important open question.

Several techniques for deriving optimality conditions are available in the literature of optimal control systems governed by partial differential equations 3, 4, 5. We obtain the optimality conditions by making use of a Lagrangian approach recently used with success by O. Bodart, A. V. Boureau and R. Touzani for an optimal control problem of the induction heating [6], and by H.-C. Lee and T. Shilkin for the thermistor problem [7.

\section{Preliminaries}

Our main objective is to obtain necessary conditions for a triple $(\bar{u}, \bar{p}, \bar{f})$ to minimize (2) among all the functions $(u, p, f)$ verifying (11). The intended necessary optimality conditions are proved in $\$ 3$ under adequate hypotheses on the data of the problem, which provide regularity of the optimal solution.

\subsection{Notation and Functional Spaces}

In the sequel we assume that $\varphi, g$ and $d$ are real valued $C^{1}$-functions satisfying:

$$
0<c_{1} \leq d(r), \varphi(r) \leq c_{2} ;\left|d^{\prime}(r)\right|,\left|\varphi^{\prime}(r)\right|,\left|\varphi^{\prime \prime}(r)\right| \leq c_{3} \quad \forall r \in \mathbb{R}
$$


(H2) $u_{0}, p_{0} \in C^{2}(\bar{\Omega}), U, P \in L^{2}\left(Q_{T}\right)$, where $u_{0}, p_{0}: \Omega \rightarrow \mathbb{R}, U, P: Q_{T} \rightarrow \mathbb{R}$, and $\left.u_{0}\right|_{\partial \Omega}=\left.p_{0}\right|_{\partial \Omega}=0$.

We consider the following spaces:

$$
W_{p}^{1,0}\left(Q_{T}\right):=L^{p}\left(0, T, W_{p}^{1}(\Omega)\right)=\left\{u \in L^{p}\left(Q_{T}\right), \nabla u \in L^{p}\left(Q_{T}\right)\right\},
$$

endowed with the norm $\|u\|_{W_{p}^{1,0}\left(Q_{T}\right)}=\|u\|_{p, Q_{T}}+\|\nabla u\|_{p, Q_{T}}$;

$$
W_{p}^{2,1}\left(Q_{T}\right):=\left\{u \in W_{p}^{1,0}\left(Q_{T}\right), \nabla^{2} u, \partial_{t} u \in L^{p}\left(Q_{T}\right)\right\},
$$

with the norm $\|u\|_{W_{p}^{2,1}\left(Q_{T}\right)}=\|u\|_{W_{p}^{1,0}\left(Q_{T}\right)}+\left\|\nabla^{2} u\right\|_{p, Q_{T}}+\left\|\partial_{t} u\right\|_{p, Q_{T}}$;

$$
\begin{gathered}
V:=\left\{u \in W_{2}^{1,0}\left(Q_{T}\right), \partial_{t} u \in L^{2}\left(0, T, W_{2}^{-1}(\Omega)\right)\right\} \\
W:=\left\{u \in W_{2 q}^{2,1}\left(Q_{T}\right),\left.u\right|_{\delta_{T}=\partial \Omega \times(0, T)}=0\right\} \\
\Upsilon:=\left\{f \in L^{2 q}\left(Q_{T}\right), \partial_{t} f \in L^{2}\left(Q_{T}\right)\right\} \\
H:=L^{2 q}\left(Q_{T}\right) \times \stackrel{\circ}{W}_{2 q}^{2-\frac{1}{q}}(\Omega) .
\end{gathered}
$$

\subsection{Coercive Estimate}

The following lemma provides a coercive estimate to linear parabolic systems that is useful for our purposes.

Lemma 2.1 ([8]). Let $\Omega$ be a bounded domain with a $C^{2}$-boundary, and assume that

$$
A_{i j k l} \in C\left(\bar{Q}_{T}\right), \quad b_{i j k} \in L^{r}\left(Q_{T}\right), \quad c_{i j} \in L^{\frac{r}{2}}\left(Q_{T}\right)
$$

with $r>n+2$ and $A$ satisfying the strong ellipticity condition:

$$
\begin{gathered}
\exists \gamma_{0}>0: A_{i j k l}(u) B_{i j} B_{k l} \geq \gamma_{0}|B|^{2} \quad \forall B \in M^{n \times n}, \\
A_{i j k l} A_{k l i j}=A_{j i k l}=A_{i j l k} .
\end{gathered}
$$

Then, for any $s \in(1, r), s \neq \frac{3}{2}$, and for arbitrary functions $f \in L^{s}\left(Q_{T}, \mathbb{R}^{n}\right)$ and $u_{0} \in \stackrel{\circ}{W}_{s}^{2-\frac{2}{s}}\left(\Omega, \mathbb{R}^{N}\right)$ there exists a unique solution $u \in W_{s}^{2,1}\left(Q_{T}, \mathbb{R}^{N}\right)$ of the problem

$$
\begin{gathered}
\partial_{t} u_{i}-A_{i j k l}(x) u_{k j l}+b_{i j k}(x) u_{j k}+c_{i j}(x) u_{j}=f_{i}(x), \\
\left.u\right|_{t=0}=u_{0},\left.\quad u\right|_{\partial \Omega}=0 .
\end{gathered}
$$

Moreover, the estimate

$$
\|u\|_{W_{s}^{2,1}\left(Q_{T}\right)} \leq c\left(\|f\|_{s, Q_{T}}+\left\|u_{0}\right\|_{W_{s}^{2-\frac{2}{s}}(\Omega)}\right)
$$

holds for some constant $c$ depending only on $n, \Omega, T, \gamma_{0}$ and the norms of the coefficients. 


\subsection{Existence of Optimal Solution}

The following existence theorem is proved in [2] using a technical lemma found in 9, Young's inequality and Aubin's Lemma, together with the theorem of Lebesgue and some compacity arguments of J. L. Lions [3]. The conclusion follows from the fact that $J$ is lower semicontinuous with respect to the weak convergence.

Theorem $2.2([2])$. Under the hypotheses (H1) and (H2) there exists a $q>$ 1 , depending on the data of the problem, such that the problem of minimizing (2) subject to (11) has an optimal solution $(\bar{u}, \bar{p}, \bar{f})$ satisfying $\bar{u} \in W_{q}^{2,1}\left(Q_{T}\right)$, $\bar{p} \in C\left([0, T] ; L^{2}(\Omega)\right) \cap W_{2 q}^{1,0}\left(Q_{T}\right), \partial_{t} \bar{p} \in L^{2}\left(0, T, W_{2}^{-1}(\Omega)\right), \bar{f} \in L^{2 q_{0}}\left(Q_{T}\right)$, $\partial_{t} \bar{f} \in L^{2}\left(Q_{T}\right)$.

\subsection{Regularity of Solutions}

Regularity of the solutions given by Theorem 2.2 is also proved in [2]. Theorem 2.3 is obtained using Young's and Holder's inequalities, Gronwall Lemma, De Giorgi-Nash-Ladyzhenskaya-Uraltseva theorem, an estimate from [10], and some technical lemmas found in [11].

Theorem $2.3([2])$. Let $(\bar{u}, \bar{p}, \bar{f})$ be an optimal solution to the problem of minimizing (2) subject to (11). Suppose that (H1) and (H2) are satisfied. Then, there exist $\alpha>0$ such that the following regularity conditions hold: $\bar{u} \in C^{\alpha, \frac{\alpha}{2}}\left(\bar{Q}_{T}\right)$,

$\bar{u}, \bar{p} \in W_{4}^{1,0}\left(Q_{T}\right), \bar{u}, \bar{p} \in W_{2}^{2,1}\left(Q_{T}\right), \partial_{t} \bar{u}, \partial_{t} \bar{p} \in L^{\infty}\left(0, T ; L^{2}(\Omega)\right) \cap W_{2}^{1,0}\left(Q_{T}\right)$, $\bar{u} \in C^{\frac{1}{4}}\left(Q_{T}\right), \bar{u} \in W_{2 q_{0}}^{2,1}\left(Q_{T}\right), \bar{p} \in W_{2 q_{0}}^{2,1}\left(Q_{T}\right)$.

\section{Main Results}

We define the following nonlinear operator corresponding to (11):

$$
\begin{gathered}
F: W \times W \times \Upsilon \longrightarrow H \times H \\
(u, p, f) \longrightarrow F(u, p, f)=0
\end{gathered}
$$

where

$$
F(u, p, f)=\left(\begin{array}{cc}
\partial_{t} u-\Delta \varphi(u)-\operatorname{div}(g(u) \nabla p), & \gamma_{0} u-u_{0} \\
\partial_{t} p-\operatorname{div}(d(u) \nabla p)-f, & \gamma_{0} p-p_{0}
\end{array}\right)
$$

$\gamma_{0}$ being the trace operator $\gamma_{0} u=\left.u\right|_{t=0}$. Owing to the estimate

$$
\|v\|_{W_{\frac{4 q}{2-q}}^{1,0}} \leq c\|v\|_{W_{2 q}^{2,1}\left(Q_{T}\right)}, \quad \forall v \in W_{2 q}^{2,1}\left(Q_{T}\right), \quad 1<q<2
$$

(see [1]) and hypothesis (H1), we have

$$
\varphi^{\prime \prime}(u)|\nabla u|^{2}, g^{\prime}(u) \nabla u \nabla p, d(u) \nabla u \nabla p \in L^{\frac{2 q}{2-q}}\left(Q_{T}\right) \subset L^{2 q}\left(Q_{T}\right) .
$$

Thus, it follows that $F$ is well defined. 


\subsection{Gâteaux differentiability}

Theorem 3.1. In addition to the hypotheses (H1) and (H2), let us suppose that

(H3) $\left|\varphi^{\prime \prime \prime}\right| \leq c$.

Then, the operator $F$ is Gâteaux differentiable and its derivative is given by

$$
\begin{aligned}
& \delta F(u, p, f)(e, w, h)=\left.\frac{d}{d s} F(u+s e, p+s w, f+s h)\right|_{s=0}=\left(\delta F_{1}, \delta F_{2}\right) \\
= & \left(\begin{array}{cc}
\partial_{t} e-\operatorname{div}\left(\varphi^{\prime}(u) \nabla e\right)-\operatorname{div}\left(\varphi^{\prime \prime}(u) e \nabla u\right)-\operatorname{div}(g(u) \nabla w)-\operatorname{div}\left(g^{\prime}(u) e \nabla p\right), & \gamma_{0} e \\
\partial_{t} w-\operatorname{div}(d(u) \nabla w)-\operatorname{div}\left(d^{\prime}(u) e \nabla p\right)-h, & \gamma_{0} w
\end{array}\right),
\end{aligned}
$$

for all $(e, w, h) \in W \times W \times \Upsilon$.

Furthermore, for any optimal solution $(\bar{u}, \bar{p}, \bar{f})$ of the problem of minimizing (2) among all the functions $(u, p, f)$ satisfying (10), the image of $\delta F(\bar{u}, \bar{p}, \bar{f})$ is equal to $H \times H$.

To prove Theorem 3.1 we make use of the following lemma.

Lemma 3.2. The operator $\delta F(u, p, f): W \times W \times \Upsilon \longrightarrow H \times H$ is linear and bounded.

Proof. of Lemma 3.2. We have for all $(e, w, h) \in W \times W \times \Upsilon$

$\delta_{p} F_{2}(u, p, f)(e, w, h)=\partial_{t} w-\operatorname{div}(d(u) \nabla w)-\operatorname{div}\left(d^{\prime}(u) e \nabla p\right)-h$

$=\partial_{t} w-d(u) \triangle w-d^{\prime}(u) \nabla u . \nabla w-d^{\prime}(u) e \Delta p-d^{\prime}(u) \nabla e . \nabla u-d^{\prime}(u) e \nabla u . \nabla p-h$,

with $\delta_{p} F$ the Gâteaux derivative of $F$ with respect to $p$. Then, using hypothesis $(H 1)$, we obtain that

$$
\begin{gathered}
\left\|\delta_{p} F_{2}(u, p, f)(e, w, h)\right\|_{2 q, Q_{T}} \leq\left\|\partial_{t} w\right\|_{2 q, Q_{T}}+\|\nabla w\|_{2 q, Q_{T}}+c\|\Delta w\|_{2 q, Q_{T}} \\
+c\|\nabla u . \nabla w\|_{2 q, Q_{T}}+c\|e \Delta p\|_{2 q, Q_{T}}+c\|\nabla e . \nabla u\|_{2 q, Q_{T}}+c\|e \nabla u . \nabla p\|_{2 q, Q_{T}}+\|h\|_{2 q, Q_{T}}
\end{gathered}
$$

In what follows we consider the term $\|e \nabla u . \nabla p\|_{2 q, Q_{T}}$. Similar arguments apply to the remaining terms of (3). We have

$$
\begin{aligned}
\|e \nabla u . \nabla p\|_{2 q, Q_{T}} & \leq\|e\|_{\infty, Q_{T}}\|\nabla u . \nabla p\|_{2 q, Q_{T}} \\
& \leq\|e\|_{\infty, Q_{T}}\|\nabla u\|_{\frac{4 q}{2-q}, Q_{T}}\|\nabla p\|_{4, Q_{T}} \\
& \leq c\|u\|_{W}\|p\|_{W}\|e\|_{W} .
\end{aligned}
$$

Then,

$$
\left\|\delta_{p} F_{2}(u, p, f)(e, w, h)\right\|_{2 q, Q_{T}} \leq c\left(\|u\|_{W},\|p\|_{W},\|f\|_{\Upsilon}\right)\left(\|e\|_{W}+\|w\|_{W}+\|h\|_{\Upsilon}\right) .
$$


On the other hand,

$$
\begin{gathered}
\delta_{u} F_{1}(u, p, f)(e, w, h)=\partial_{t} e-\operatorname{div}\left(\varphi^{\prime}(u) \nabla e\right)-\operatorname{div}\left(\varphi^{\prime \prime}(u) e \nabla u\right) \\
-\operatorname{div}(g(u) \nabla w)-\operatorname{div}\left(g^{\prime}(u) e \nabla p\right) \\
=\partial_{t} e-\varphi^{\prime}(u) \triangle e-\varphi^{\prime \prime}(u) \nabla u . \nabla e \\
-\varphi^{\prime \prime}(u) e \Delta u-\varphi^{\prime \prime}(u) \nabla e . \nabla u-\varphi^{\prime \prime \prime}(u) e|\nabla u|^{2} \\
-g(u) \triangle w-g^{\prime}(u) \nabla u . \nabla w \\
-g^{\prime}(u) e \triangle p-g^{\prime}(u) \nabla e . \nabla p-g^{\prime \prime}(u) e \nabla u . \nabla p,
\end{gathered}
$$

where $\delta_{u} F$ is the Gâteaux derivative of $F$ with respect to $u$. The same argument as above give that

$$
\left\|\delta_{u} F_{1}(u, p, f)(e, w, h)\right\|_{2 q, Q_{T}} \leq c\left(\|u\|_{W},\|p\|_{W},\|f\|_{\Upsilon}\right)\left(\|e\|_{W}+\|w\|_{W}+\|h\|_{\Upsilon}\right) .
$$

Consequently, by (4) and (5) we can write

$\|\delta F(u, p, f)(e, w, h)\|_{H \times H \times \Upsilon} \leq c\left(\|u\|_{W},\|p\|_{W},\|f\|_{\Upsilon}\right)\left(\|e\|_{W}+\|w\|_{W}+\|h\|_{\Upsilon}\right)$.

Proof. of Theorem 3.1 In order to show that the image of $\delta F(\bar{u}, \bar{p}, \bar{f})$ is equal to $H \times H$, we need to prove that there exists a $(w, e, h) \in W \times W \times \Upsilon$ such that

$$
\begin{gathered}
\partial_{t} e-\operatorname{div}\left(\varphi^{\prime}(\bar{u}) \nabla e\right)-\operatorname{div}\left(\varphi^{\prime \prime}(\bar{u}) e \nabla \bar{u}\right)-\operatorname{div}(g(\bar{u}) \nabla w)-\operatorname{div}\left(g^{\prime}(\bar{u}) e \nabla \bar{p}\right)=\alpha, \\
\partial_{t} w-\operatorname{div}(d(\bar{u}) \nabla w)-\operatorname{div}\left(d^{\prime}(\bar{u}) e \nabla \bar{p}\right)-h=\beta, \\
\left.e\right|_{\partial \Omega}=0,\left.\quad e\right|_{t=0}=b \\
\left.w\right|_{\partial \Omega}=0,\left.\quad w\right|_{t=0}=a
\end{gathered}
$$

for any $(\alpha, a)$ and $(\beta, b) \in H$. Writing the system (6) for $h=0$ as

$$
\begin{gathered}
\partial_{t} e-\varphi^{\prime}(\bar{u}) \triangle e-2 \varphi^{\prime \prime}(\bar{u}) \nabla \bar{u} . \nabla e-\varphi^{\prime \prime}(\bar{u}) e \triangle \bar{u}-\varphi^{\prime \prime \prime}(\bar{u}) e|\nabla \bar{u}|^{2}, \\
-g(\bar{u}) \triangle w-g^{\prime}(\bar{u}) \nabla \bar{u} . \nabla w-g^{\prime}(\bar{u}) e \triangle \bar{p}-g^{\prime}(\bar{u}) \nabla \bar{p} . \nabla e-g^{\prime \prime}(\bar{u}) e \nabla \bar{u} . \nabla \bar{p}=\alpha, \\
\partial_{t} w-d(\bar{u}) \triangle w-d^{\prime}(\bar{u}) \nabla \bar{u} . \nabla w-d^{\prime}(\bar{u}) e \triangle \bar{p}-d^{\prime}(\bar{u}) \nabla \bar{u} . \nabla \bar{e}-d^{\prime}(\bar{u}) e \nabla \bar{u} . \nabla \bar{p}=\beta, \\
\left.e\right|_{\partial \Omega}=0,\left.\quad e\right|_{t=0}=b, \\
\left.w\right|_{\partial \Omega}=0,\left.\quad w\right|_{t=0}=a,
\end{gathered}
$$

it follows from the regularity of the optimal solution (Theorem 2.3) that

$$
\begin{gathered}
\varphi^{\prime \prime}(\bar{u}) \triangle \bar{u}, \varphi^{\prime \prime \prime}(\bar{u})|\nabla \bar{u}|^{2}, g^{\prime}(\bar{u}) \triangle \bar{p}, g^{\prime \prime}(\bar{u}) \nabla \bar{u} . \nabla \bar{p}, d^{\prime}(\bar{u}) \triangle \bar{p}, d^{\prime}(\bar{u}) \nabla \bar{u} . \nabla \bar{p} \in L^{2 q_{0}\left(Q_{T}\right)}, \\
\varphi^{\prime \prime}(\bar{u}) \nabla \bar{u}, g^{\prime}(\bar{u}) \nabla \bar{u}, g^{\prime}(\bar{u}) \nabla \bar{p}, d^{\prime}(\bar{u}) \nabla \bar{u} \in L^{4 q_{0}}\left(Q_{T}\right) .
\end{gathered}
$$

By Lemma 2.1 there exists a unique solution of the system (77), hence there exists a $(e, w, 0)$ verifying (6). We conclude that the image of $\delta F$ is equal to $H \times H$. 


\subsection{Necessary Optimality Condition}

We consider the cost functional $J: W \times W \times \Upsilon \rightarrow \mathbb{R}(2)$ and the Lagrangian $\mathcal{L}$ defined by

$$
\mathcal{L}\left(u, p, f, p_{1}, e_{1}, a, b\right)=J(u, p, f)+\left\langle F(u, p, f),\left(\begin{array}{cc}
p_{1} & a \\
e_{1}, & b
\end{array}\right)\right\rangle,
$$

where the bracket $\langle\cdot, \cdot\rangle$ denote the duality between $H$ and $H^{\prime}$.

Theorem 3.3. Under hypotheses (H1)-(H3), if $(\bar{u}, \bar{p}, \bar{f})$ is an optimal solution to the problem of minimizing (2) subject to (1), then there exist functions $\left(\overline{e_{1}}, \overline{p_{1}}\right) \in W_{2}^{2,1}\left(Q_{T}\right) \times W_{2}^{2,1}\left(Q_{T}\right)$ satisfying the following conditions:

$$
\begin{gathered}
\partial_{t} \overline{e_{1}}+\operatorname{div}\left(\varphi^{\prime}(\bar{u}) \nabla e_{1}\right)-d^{\prime}(\bar{u}) \nabla \bar{p} . \nabla \overline{p_{1}}-\varphi^{\prime \prime}(\bar{u}) \nabla \bar{u} . \nabla \overline{e_{1}}-g^{\prime}(\bar{u}) \nabla \bar{p} . \nabla \overline{e_{1}}=\bar{u}-U \\
\left.\overline{e_{1}}\right|_{\partial \Omega}=0,\left.\quad \overline{e_{1}}\right|_{t=T}=0 \\
\partial_{t} \overline{p_{1}}+\operatorname{div}\left(d(\bar{u}) \nabla \overline{p_{1}}\right)+\operatorname{div}\left(g(\bar{u}) \nabla \overline{e_{1}}\right)=\bar{p}-P \\
\left.\overline{p_{1}}\right|_{\partial \Omega}=0,\left.\quad \overline{p_{1}}\right|_{t=T}=0 \\
-\beta_{2} \frac{\partial^{2} \bar{f}}{\partial t^{2}}+2 q_{0} \beta_{1}|\bar{f}|^{2 q_{0}-2} \bar{f}=\overline{p_{1}},\left.\quad \frac{\partial \bar{f}}{\partial t}\right|_{t=0}=\left.\frac{\partial \bar{f}}{\partial t}\right|_{t=T}=0
\end{gathered}
$$

Proof. Let $(\bar{u}, \bar{p}, \bar{f})$ be an optimal solution to the problem of minimizing (2) subject to (11). It is well known (cf. e.g. [12]) that there exist Lagrange multipliers $\left(\left(\overline{p_{1}}, \bar{a}\right),\left(\overline{e_{1}}, \bar{b}\right)\right) \in H^{\prime} \times H^{\prime}$ verifying

$$
\delta_{(u, p, f)} \mathcal{L}\left(\bar{u}, \bar{p}, \bar{f}, \overline{p_{1}}, \overline{e_{1}}, \bar{a}, \bar{b}\right)(e, w, h)=0 \quad \forall(e, w, h) \in W \times W \times \Upsilon,
$$

with $\delta_{(u, p, f)} \mathcal{L}$ the Gâteaux derivative of $\mathcal{L}$ with respect to $(u, p, f)$. We then obtain

$$
\begin{gathered}
\int_{Q_{T}}\left((\bar{u}-U) e+(\bar{p}-P) w+2 q_{0} \beta_{1}|\bar{f}|^{2 q_{0}-2} \bar{f} h+\beta_{2} \partial_{t} \bar{f} \partial_{t} h\right) d x d t \\
-\int_{Q_{T}}\left(\partial_{t} e-\operatorname{div}\left(\varphi^{\prime}(\bar{u}) \nabla e\right)-\operatorname{div}\left(\varphi^{\prime \prime}(\bar{u}) e \nabla \bar{u}\right)-\operatorname{div}(g(\bar{u}) \nabla w)-\operatorname{div}\left(g^{\prime}(\bar{u}) e \nabla \bar{p}\right)\right) \overline{e_{1}} d x d t \\
-\int_{Q_{T}}\left(\partial_{t} w-\operatorname{div}(d(\bar{u}) \nabla w)-\operatorname{div}\left(d^{\prime}(\bar{u}) e \nabla \bar{p}\right)-h\right) \overline{p_{1}} d x d t \\
-\left\langle\gamma_{0} e, \bar{a}\right\rangle+-\left\langle\gamma_{0} w, \bar{b}\right\rangle=0 \quad \forall(e, w, h) \in W \times W \times \Upsilon .
\end{gathered}
$$

This last system is equivalent to the following one:

$$
\begin{aligned}
& \int_{Q_{T}}\left((\bar{u}-U) e-\operatorname{div}\left(d^{\prime}(\bar{u}) e \nabla \bar{p}\right) \overline{p_{1}}+\partial_{t} e \overline{e_{1}}-\operatorname{div}\left(\varphi^{\prime}(\bar{u}) \nabla e\right) \overline{e_{1}}\right. \\
& \left.-\operatorname{div}\left(\varphi^{\prime \prime}(\bar{u}) e \nabla \bar{u}\right) \overline{e_{1}}-\operatorname{div}\left(g^{\prime}(\bar{u}) e \nabla \bar{p}\right) \overline{e_{1}}\right) d x d t \\
& +\int_{Q_{T}}\left((\bar{p}-P) w+\partial_{t} w \overline{p_{1}}-\operatorname{div}(d(\bar{u}) \nabla w) \overline{p_{1}}-\operatorname{div}(g(\bar{u}) \nabla w) \overline{e_{1}}\right) d x d t \\
& +\int_{Q_{T}}\left(2 q_{0} \beta_{1}|\bar{f}|^{2 q_{0}-2} \bar{f} h+\beta_{2} \partial_{t} \bar{f} \partial_{t} h-\overline{p_{1}} h\right) d x d t \\
& +\left\langle\gamma_{0} e, \bar{a}\right\rangle+\left\langle\gamma_{0} w, \bar{b}\right\rangle=0 \quad \forall(e, w, h) \in W \times W \times \Upsilon .
\end{aligned}
$$


In others words, we have

$$
\begin{gathered}
\int_{Q_{T}}\left((\bar{u}-U)+d^{\prime}(u) \nabla \bar{p} . \nabla \overline{p_{1}}-\partial_{t} \overline{e_{1}}-\operatorname{div}\left(\varphi^{\prime}(\bar{u}) \nabla \overline{e_{1}}\right)+\varphi^{\prime \prime}(\bar{u}) \nabla \bar{u} \cdot \nabla \overline{e_{1}}+g^{\prime}(u) \nabla \bar{p} . \nabla \overline{e_{1}}\right) e d x d t \\
+\int_{Q_{T}}\left((\bar{p}-P)+\partial_{t} \overline{p_{1}}-\operatorname{div}\left(d(\bar{u}) \nabla \overline{p_{1}}\right)-\operatorname{div}\left(g(\bar{u}) \nabla \overline{e_{1}}\right)\right) w d x d t \\
+\int_{Q_{T}}\left(2 q_{0} \beta_{1}|\bar{f}|^{2 q_{0}-2} \bar{f} h+\beta_{2} \partial_{t} \bar{f} \partial_{t} h-\overline{p_{1}} h\right) d x d t \\
+\left\langle\gamma_{0} e, \bar{a}\right\rangle+\left\langle\gamma_{0} w, \bar{b}\right\rangle=0 \forall(e, w, h) \in W \times W \times \Upsilon .
\end{gathered}
$$

Consider now the system

$$
\begin{gathered}
\partial_{t} e_{1}+\operatorname{div}\left(\varphi^{\prime}(\bar{u}) \nabla e_{1}\right)-d^{\prime}(\bar{u}) \nabla \bar{p} . \nabla p_{1}-\varphi^{\prime \prime}(\bar{u}) \nabla \bar{u} . \nabla e_{1}-g^{\prime}(\bar{u}) \nabla \bar{p} . \nabla e_{1}=\bar{u}-U \\
\partial_{t} p_{1}+\operatorname{div}\left(d(\bar{u}) \nabla p_{1}\right)+\operatorname{div}\left(g(\bar{u}) \nabla e_{1}\right)=\bar{p}-P \\
\left.e_{1}\right|_{\partial \Omega}=\left.p_{1}\right|_{\partial \Omega}=0,\left.\quad e_{1}\right|_{t=T}=\left.p_{1}\right|_{t=T}=0
\end{gathered}
$$

It follows by Lemma 2.1 that (10) has a unique solution $\left(e_{1}, p_{1}\right) \in W_{2}^{2,1}\left(Q_{T}\right) \times$ $W_{2}^{2,1}\left(Q_{T}\right)$. Since the problem of finding $(e, w) \in W \times W$ satisfying

$$
\begin{gathered}
\partial_{t} e-\operatorname{div}\left(\varphi^{\prime}(\bar{u}) \nabla e\right)-\operatorname{div}\left(\varphi^{\prime \prime}(\bar{u}) e \nabla \bar{u}\right)-\operatorname{div}(g(\bar{u}) \nabla w)-\operatorname{div}\left(g^{\prime}(\bar{u}) e \nabla \bar{p}\right)=\operatorname{sign}\left(e_{1}-\overline{e_{1}}\right) \\
\partial_{t} w-\operatorname{div}(d(\bar{u}) \nabla w)-\operatorname{div}\left(d^{\prime}(\bar{u}) e \nabla \bar{p}\right)=\operatorname{sign}\left(p_{1}-\overline{p_{1}}\right) \\
\gamma_{0} e=\gamma_{0} w=0
\end{gathered}
$$

is uniquely solvable on $W_{2 q}^{2,1} \times W_{2 q}^{2,1}$ by Lemma 2.1 choosing $h=0$ in (9), multiplying (10) by $(e, w)$, integrating by parts, and making the difference with (9), we obtain

$$
\begin{gathered}
\int_{Q_{T}}\left(\partial_{t} e-\operatorname{div}\left(\varphi^{\prime}(\bar{u}) \nabla e\right)-\operatorname{div}\left(\varphi^{\prime \prime}(\bar{u}) e \nabla \bar{u}\right)-\operatorname{div}(g(\bar{u}) \nabla w)-\operatorname{div}\left(g^{\prime}(\bar{u}) e \nabla \bar{p}\right)\right)\left(e_{1}-\overline{e_{1}}\right) d x d t \\
+\int_{Q_{T}}\left(\partial_{t} w-\operatorname{div}(d(\bar{u}) \nabla w)-\operatorname{div}\left(d^{\prime}(\bar{u}) e \nabla \bar{p}\right)\right)\left(p_{1}-\overline{p_{1}}\right) d x d t \\
+\left\langle\gamma_{0} e, \gamma_{0} \overline{e_{1}}-\bar{a}\right\rangle+\left\langle\gamma_{0} w, \gamma_{0} \overline{p_{1}}-\bar{b}\right\rangle=0 \quad \forall(e, w) \in W \times W .
\end{gathered}
$$

Choosing $(e, w)$ in (12) as the solution of the system (11), we have

$$
\int_{Q_{T}} \operatorname{sign}\left(e_{1}-\overline{e_{1}}\right)\left(e_{1}-\overline{e_{1}}\right) d x d t+\int_{Q_{T}} \operatorname{sign}\left(p_{1}-\overline{p_{1}}\right)\left(p_{1}-\overline{p_{1}}\right) d x d t=0 .
$$

It follows that $e_{1}=\overline{e_{1}}$ and $p_{1}=\overline{p_{1}}$. Coming back to (12), we obtain $\gamma_{0} \overline{e_{1}}=\bar{a}$ and $\gamma_{0} \overline{p_{1}}=\bar{b}$. On the other hand, choosing $(e, w)=(0,0)$ in (9) it follows (8), which conclude the proof of Theorem 3.3 


\section{References}

[1] GAGNEUX, G., and MADAUNE-TORT, M., Analyse mathématique de modèles non linéaires de l'ingénierie pétrolière, Mathématiques \& Application 22, Springer-Verlag, Berlin, 1996.

[2] SIDI AMMI, M. R., and TORRES, D. F. M., Existence and regularity of optimal solution for a dead oil isotherm problem, Differential GeometryDynamical Systems, Vol. 9, No. 1, 2007 (electronic).

[3] LIONS, J.-L., Quelques méthodes de résolution des problèmes aux limites non linéaires, Dunod, Paris, 1969.

[4] LIONS, J.-L., Optimal control of systems governed by partial differential equations, Translated from the French by S. K. Mitter. Die Grundlehren der mathematischen Wissenschaften, Springer, New York, 1971.

[5] MORDUKHOVICH, B. S., Variational analysis and generalized differentiation II-Applications, Springer, Berlin, 2006.

[6] BODART, O., BOUREAU, A. V., and TOUZANI, R., Numerical investigation of optimal control of induction heating processes, Applied Mathematical Modelling, Vol. 25, pp. 697-712, 2001.

[7] LEE, H.-C., and SHILKIN, T., Analysis of optimal control problems for the two-dimensional thermistor system, SIAM Journal on Control and Optimization, Vol. 44, No. 1, pp. 268-282, 2005.

[8] SOLONNIKOV, V. A., On boundary value problems for linear parabolic systems of differential equations of general form, Trudy Matematicheskogo Instituta imeni V. A. Steklova, Vol. 83, pp. 3-163, 1965.

[9] Bensoussan, A., LIONS, J. L., and PAPAnICOLAOU, G., Asymptotic Analysis for periodic structures, Studies in Mathematics and its Applications, 5. North-Holland Publishing Co., Amsterdam-New York, 1978.

[10] KOCH, H., SOLONNIKOV, V. A., L $L_{p}$-estimates of solutions of the nonstationary stokes problem, Journal of Mathematical Sciences (New York), Vol. 106, pp. 3042-3072, 2001.

[11] LADYZHENSKAYA, O. A., SOLONNIKOV, V. A., and URALTSEVA, N. N., Linear and quasi-linear equations of parabolic type, Transl. Math. Monogr. 23, AMS, Providence, RI, 1967.

[12] FURSIKOV, A. V., Optimal control of distributed systems, Theory and applications, AMS, Providence, RI, 2000. 